\title{
Acknowledgment to Reviewers of Social Sciences in 2021
}

\author{
Social Sciences Editorial Office
}

Citation: Social Sciences Editorial Office. 2022. Acknowledgment to Reviewers of Social Sciences in 2021.

Social Sciences 11: 45. https://doi.org/ 10.3390/socsci11020045

Published: 27 January 2022

Publisher's Note: MDPI stays neutral with regard to jurisdictional claims in published maps and institutional affiliations.

Copyright: (C) 2022 by the author. Licensee MDPI, Basel, Switzerland. This article is an open access article distributed under the terms and conditions of the Creative Commons Attribution (CC BY) license (https://creativecommons.org/license s/by/4.0/).

MDPI AG, St. Alban-Anlage 66, 4052 Basel, Switzerland

Rigorous peer-reviews are the basis of high-quality academic publishing. Thanks to the great efforts of our reviewers, Social Sciences was able to maintain its standards for the high quality of its published papers. Thanks to the contribution of our reviewers, in 2021, the median time to first decision was 25 days and the median time to publication was 54 days. The editors would like to extend their gratitude and recognition to the following reviewers for their precious time and dedication, regardless of whether the papers they reviewed were finally published:

Abbondanza, Gabriele

Abduljaber, Malek

Abel, Gillian

Acevedo, Jesse

Adamski, Andrzej

Addeo, Felice

Agarin, Timofey

Aguaded-Ramírez, Eva

Aguilar, Miguel Pic

Ahmad, Jared

Akhter, Rifat

Albastroiu, Irina

Al-Kassimi, Khaled

Alonso, Francisco

Alonso-Muñoz, Laura

Al-Rahmi, Waleed

Altwaiji, Mubarak

Álvarez-Serrano, María Adelaida

Amâncio, Lígia

Amor Almedina, María Isabel

Amor González, Antonio Manuel

Anastasiou, Evgenia

Anderson, Sharon

Angelidou, Georgia

Antia, Khatia

Arcangeli, Giulio

Ares, Alberto

Arias, Ana Victoria

Arjona, Joaquín Osorio

Armstrong, Helen

Arnold, Bruce Baer

Asad, Asad L.

Atilgan, Hatice

Auma, Maisha Maureen

Avdimiotis, Spyros
Aziz Ur Rehman, Syed

Badreldin, Ahmed Mohamed

Badulescu, Alina

Bagley, Christopher Adam

Bagwell, Stephen

Bairner, Alan

Baker-Beall, Christopher

Baldwin, John

Bándy, Katalin

Bănică, Alexandru

Baranowska, Barbara

Barbero, Iker

Barcik, Jacek

Barn, Ravinder

Barrera, Alinne Z.

Barros, Paula

Bartone, Paul

Bartus, Tamás

Baruffini, Moreno

Baumann, Chris

Bell, Adrian

Bell, Simon

Bellia, Claudio

Benčina, Jože

Benjamin, Elliot

Bennachie, Calum

Berei, Emese Beáta

Berezuk, André Geraldo

Bernadowski, Carianne

Bernasek, Lisa

Besalu, Reinald

Bethem, Jacob

Betz, Hans-Georg

Bianco, Rosella

Bilan, Svitlana 
Bilbao, Naiara

Binnema, Harmen

Blakey, Joan

Blanton, Robert

Blasco, Yolanda

Bligh, Brett

Blue, Sarah

Boeren, Ellen

Bogoslov, Ioana Andreea

Bohle, Martin

Bolz, Daphné

Bonefeld, Werner

Bongelli, Ramona

Bonino, Stefano

Bonneau, Josée

Bopp, Judith

Bordon, Paola

Borgerhoff Mulder, Monique

Borges, Susana

Borzikowsky, Christoph

Boscaljon, Daniel

Bostenaru Dan, Maria

Bouma, Gary

Boyd, Robert

Brand, Serge

Brandariz García, José Ángel

Brandariz, José A.

Braunbeck, Helga G.

Broad, Rose

Broadhead, Samantha

Brocchi, Beatriz Servilha

Broglia, Emma

Bronikowski, Michał

Bronzaft, Arline

Brown Guzmán, Danice

Brown, Julianna

Bukovčan, Tanja

Bulkeley, Kim

Bunge, Eduardo

Byon, Kevin K.

Cabrera-Barona, Pablo

Caines, Valerie

Cairns, David

Calvo, Dafne

Camorrino, Antonio

Campbell, Janine Anne

Campion, Kristy

Cañabate, Dolors

Canário, Catarina

Cantero-Garlito, Pablo A.

Card, Kiffer
Caridade, Sónia Maria Martins

Carr, James

Carrillo Murcia, Irene

Carrillo-Álvarez, Elena

Carroll, Páraic

Carson, Dena

Caspe, Margaret

Cassidy, Tony

Castellví, Jordi

Castillo Apraiz, Julen

Cavignac, Julie

Cerón, Alejandro

Česnakas, Giedrius

Chalmers, Darlene

Chandler, Nick

Chang, Poju

Chao, Fengqing

Cheek, Wesley W.

Chen, Ru-Si

Chen, Si-Tong

Chi, Ting

Chigbu, Uchendu Eugene

Choate, Peter W.

Choi, Jun H.

Cieślińska, Barbara

Cingranelli, David

Clare, Stephanie

Clark, Mitch

Co Sy Su, Chadwick

Colombo, Barbara

Comes, Calin-Adrian

Constantin, Sandra

Constantinou, Costas S.

Cooney, Teresa M.

Cornelius Smith, Erika

Cortés, Juve J.

Costa, Bruno

Cotfas, Liviu-Adrian

Cowe, Jennifer

Cradock, Gerald

Crespi, Isabella

Cretan, Remus

Cristófol Rodríguez, Carmen

Cuccaro-Alamin, Stephanie

Cuenca López, José María

Cuthbertson, Joseph

Cutrona, Carolyn

Czyżowska, Dorota

Dąbrowski, Karol

Dakessian, Arek

Dalu, Mwazvita T. B. 
Daroń, Marta

Dávid-Barrett, Tamás

de Moraes Prata Gaspar, Maria Clara

De Pree, Thomas A.

Delcea, Camelia

Delgado-Algarra, Emilio J.

Dennison, Christopher

Densley, James

DeVance Taliaferro, Jocelyn

Di Nuovo, Santo

Di Sarno, Marco

Díaz- Noci, Javier

Díaz Noguera, María Dolores

Diez Bosch, Miriam

Díez-Bedmar, María Del Consuelo

Dimitrova, Elitsa

Dingil, Ali Enes

Diogo, Elisete

Dirakis, Alexis

Domaradzki, Jan

Dominguez Folgueras, Marta

Donevant, Sara B.

Donkor, Prince

Dos Santos Pereira, Fernanda Maria

Dragan, Bogdan

Drechselová, Lucie

Druen, Perri

Druica, Elena

Dueñas, Jorge-Manuel

Dunbar, Adam

Dundes, Lauren

Dworkin, Jodi

Dwyer, Angela

Ehwi, Richmond

Elkordy, Angela

Ellis, Basia

Emmott, Emily

Engeman, Cassandra

Enriquez, Laura E.

Eriksson, Anna

Escandón, Pablo

Esposito De Falco, Salvatore

Esposito, Gianluca

Esteban-Figuerola, Patricia

Estellés, Marta

Evers, Adalbert

Ewart, Jacqueline

Eylon, Yuval

Fahmy, Chantal

Fáima, Gameiro

Falcone, Pasquale Marcello
Famoso, Fabio

Fawcett, Barbara

Felknor, Sarah A.

Fellendorf, Frederike T.

Feniger, Yariv

Fernandes, Emília

Fernandes, Sandra Raquel Gonçalves

Fernández Carrasco, Francisco Javier

Fernández-Martínez, María Del Mar

Fernández-Prados, Juan Sebastián

Ferrara, Pietro

Ferreira, Jorge Manuel Leitão

Fischetti, John

Fladvad, Benno

Flanders-Crosby, Jill

Fleisher, Mark

Flinn, Mark

Frideres, James

Frumkin, Bruce

Gabel, Shirley Gatenio

Gall, Robert

Gamidullaeva, Leyla A.

Gamondi, Claudia

García, Oscar

Garcia-Fuentes, Pablo A.

García-Martínez, Inmaculada

García-Mendoza, María

Gareau, Brian J.

Gauxachs, Alba Sabaté

Gawel, Aleksandra

Geerts, Robbe

Gellman, Mneesha

Gerson, Sheri

Ghimire, Jiwnath

Gierczyk, Marcin

Gierycz, Michał

Gillies, Robyn

Gil-Salmerón, Alejandro

Giménez-Llort, Lydia

Glässel, Andrea

Gogonas, Nikolaos

Gómez Cervantes, Andrea

Gómez-Berrocal, Carmen

Gonzalez Lopez, Maria Jose

Gonzálvez-Vallés, Juan Enrique

Graham, William

Griffiths, Laura

Grumi, Serena

Guss, Shannon

Gwiaździńska-Goraj, Marta

Haden, Sara Chiara 
Häkli, Jouni

Halilovich, Hariz

Hameršak, Marijana

Hames, Raymond

Harper, Frances K.

Harris, Jasmine

Harton, Anna

Hategan, Vasile

Hedges, Jerris

Henriksson, Helena Wahlström

Herman, Emilia

Hinch, Ronald

Hintjens, Helen

Hoang, Linh N.

Hodgson, Ian

Hoffstaedter, Gerhard

Hom, Laureen

Hopcroft, Rosemary

Hopkinson, Leo

Hörnsten, Åsa

Howard, Janet

Howell, Jayne

Hugelius, Karin

Hughes, Michael

Hysa, Beata

Ignatowski, Grzegorz

Indiana, Maria Luisa

Ingram, Erin

Irving, Justin

Jackson, Richard

Jäger, Johannes

Janowski, Andrzej

Jardim, Jacinto

Jeffrey, Leslie

Jensen, Kari B.

Jeynes, William

Jiménez, Lucía

Jochnowitz, Leona Deborah

Johanson, Jan-Erik

Jonasson, Kalle

Jones, David

Jones, Melanie

Jordan, Zoe

Jorge-Vázquez, Javier

Jovarauskaite, Lina

Jowsey, Tanisha

Jukan, Meldina Kokorović

Jukneviciene, Vita

Jung, Jin-Heon

Kalinowski, Sławomir

Kaltsas, Spyridon
Kania, Aneta

Karampelas, Konstantinos

Karandikar, Sharvari

Katchanovski, Ivan

Kaunert, Christian

Kefalis, Chrysovalantis

Keirns, Natalie

Kendall-Taylor, Nathaniel

Khairi, Aizat

Khan, Alamgir

Kim, Hyun-Duck

Kim, Kyoung-Yim

Kim, Sang-Joon

Kimihiko, Hyakumura

Kirkwood, Steve

Kitagawa, Kaori

Kivimaki, Timo

Kivimäki, Timo

Klaus, Witold

Knapman, Gareth

Knight, David S.

Knight, Thomas Daniel

Kobeissy, Firas

Kodama, Tomoko

Kolnhofer Derecskei, Anita

Kołodziejczak, Małgorzata

Konhäusner, Peter

Konstantinov, Vsevolod V.

Kooi, Brandon

Koskela, Tarja

Koster, Jeremy

Kostoulas-Makrakis, Nelly

Kras, Kimberly

Krase, Jerome

Krysta, Krzysztof

Kulic, Nevena

Kumar BATAR, Amit

Kutsar, Dagmar

Kwan, Christine Manlai

Laka, Jon Paul

Lan, Minxuan

Langlais, Michael

Larsson, Göran

Lawson, David

Leath, Seanna

Lee, Chang Won

Lee, David

Lee, Hyoung Suk

Lee, Wai Sum

Lesyk, Lilia

Lewis, John 
Lewis, Philip

Li, Peter Ping

Li, Qirui

Liberska, Hanna

Lidén, Hilde

Lifshitz, Isaac

Lim, Lyndon

Lin, Hsiao-Hsien

Lindeman, Sari

Lindvall, Kristina

Lis, Adriana

Lloret, Antonio

Lo Giudice, Alessio

Lo Storto, Corrado

Loia, Francesca

Long, Emily

Long, Maureen

Lopes, João M.

López Fernández, José Antonio

López-Borrull, Alexandre

López-Cordero, Rafael

López-Fernández, Jorge

López-Martínez, Gabriel

Lopez-Meri, Amparo

López-Morales, Virgilio

Lordan, Ronan

Lorenz, Walter August

Lounasmaa, Aura

Lovik, Anikó

Lu, Fan

Lucarelli, Stefano

Luh, Yir-Hueih

Luhr, Sigrid

Lukesch, Robert

Luo, Yi

Luong, Hai

Lupu-Dima, Lucian

Łuszczki, Edyta

Lutkiewicz, Karolina

Lyon-Callo, Vincent

MacKenzie, Alexander

Mackinem, Mitchell

Majic, Samantha

Makore, Busisiwe Chikomborero Ncube

Mallion, Jaimee

Mallon, Benjamin

Mamen, Asgeir

Mancinas Chávez, Rosalba

Mancino, M. Antonella

Manzoni, Anna

Marbaniang, Domenic
Marceau, Justin F.

Marcellini, Anne

Marcos García, Silvia

Marcu, Silvia

Marhavilas, Panagiotis K.

María Reina, Ferrández Berrueco

Marinova, Zoya

Markowska-Manista, Urszula

Martin Cervantes, Pedro A.

Martín, J.E. Jamal

Martin, Karin D.

Martínez Lirola, María

Martínez Ramón, Juan Pedro

Martínez-Rico, Gabriel

Martini, Mara

Martins, Cátia

Mat Piah, Mohd Bijarimi

Matosas-López, Luis

Matthews, Todd L.

Matuzeviciute, Kristina

Maylor, Uvanney

Mazilescu, Crisanta-Alina

Mazur, Joanna

Mazzucato, Valentina

McAslan, Devon

McLoughlin, Claire

McNeil-Willson, Richard

McNultyt, Thomas L.

Medina García, Marta

Meek, Jack W.

Mendes, Ireneu

Méndez Mateo, Inmaculada

Metcalf, Jacob

Micallef, Nicholas

Miciuła, Ireneusz

Micó Sanz, Josep Lluís

Miley, Thomas Jeffrey

Minichiello, Angela

Minichiello, Victor

Missbach, Antje

Mitchell, Gregory

Moffette, David

Moldovan, Octavian

Molina Rodríguez-Navas, Pedro

Molina-Torres, María-Pilar

Monaco, Salvatore

Monnot, Christophe

Montazer, Shirin

Montoya, T. Mark

Moore, Tim

Moreno Molina, David 
Moreno-Marcos, Pedro Manuel

Moreno-Vera, Juan-Ramón

Morita, Masahito

Morris, Edward

Morrison, Todd G.

Moscatelli, Silvia

Motyka, Marek

Mpofu, Charles

Mucci, Nicola

Mukuni, Joseph

Muñoz, Isidro Sepulveda

Muñoz-Bermejo, Laura

Muskin, Philip R.

Mustosmäki, Armi

Myers, Samuel

Nackerud, Larry

Nafees, Lubna

Naidoo, Keshrie

Nam, Taewoo

Naples, Lauren H.

Närvi, Johanna

Nathans, Laura

Naumov, Nikola

Navarro, Raul

Nemeth, Karoly

Neocleous, Gregory

Nepal, Smriti

Nepeina, Kseniia

Newson, Lesley

Nicholls, Christine

Nicolas, Claire

Niort, Jannick

Obioha, Emeka E.

Ochoa Jiménez, Sergio

Odasso, Laura

Okada, Isamu

Okanović, Andrea

Okeke-Ihejirika, Philomina

Okręglicka, Małgorzata

Okubo, Yuko

Oliveira, Márcio

Omair, Muhammad

Oncioiu, Ionica

Onder, Mehmet

Ongena, Guido

Oselin, Sharon S.

Otero-López, José Manuel

Owen, Stephen

Ozanne, Lucie Kathleen

Özkan, Doruk Görkem

Pablo-Martí, Federico
Page, Abigail

Palidda, Salvatore

Papadopoulou, Penelope

Papageorgiou, Dimitris

Paparusso, Angela

Pasculli, Lorenzo

Pasha, Talaat

Pastor Pérez, Ana

Pastor Seller, Enrique

Pauluzzo, Rubens

Pawlak, Karolina

Pazhoohi, Farid

Pearce, Jenny

Peck, Jennifer

Pedersen, Daphne E.

Peña-Longobardo, Luz María

Peng, Michael Yao-Ping

Penman, Will

Pereira, Dora

Pérez-Curiel, Concha

Peric, Marko

Perkumienè, Dalia

Pernilla, Myrne

Pester, Andreas

Pfeiffer, Christian

Philalithis, Anastas

Pieńkowska-Kamieniecka, Sylwia

Pierre, André

Pina, David

Piñeiro, Isabel

Pintassilgo, Sónia Cardoso

Pinto Ferreira, Luís Carlos

Piosik, Andrzej

Piotrowski, Andrzej

Pippi, Roberto

Pitti, Julio Alvarez

Plopeanu, Aurelian-Petrus,

Plyushteva, Anna

Pomianek, Iwona

Poole, Jay

Popa, Daniela

Posso, Alberto

Power, Eleanor A.

Pranckutè, Raminta

Prior, Sarah

Punziano, Gabriella

Putnam, Samuel

Qasim, Salma

Quam-Wickham, Nancy

Quintana-Murci, Elena

Radcliffe, John 
Ragazou, Konstantina

Räisänen, Heikki

Rakowska, Joanna

Rania, Nadia

Rapoport, Anatoli

Ratajczak, Magdalena

Raya, Antonio Martínez

Redhead, Daniel

Reksten, Nicholas

Rennó Santos, Mateus

Revez, Jorge

Rhee, Seung-Yoon

Richert-Kaźmierska, Anita

Ridley, Josephine

Rizun, Mariia

Rodríguez Jiménez, Carmen

Rodríguez-Fernández, Juan R.

Rodríguez-Martín, Alejandro

Rodriguez-Modroño, Paula

Rodríguez-Sánchez, José-Luis

Rogobete, Silviu

Ronsivalle, Daniele

Rosa Gonzalez-Martinez, Ana

Rosak-Szyrocka, Joanna

Rosasco, Paolo

Rössl, Lydia Theresia

Roth, Adam

Roussos, Sotiris

Rowley, Georgia

Rozario, Sophia Diana

Ruban, Dmitry A.

Ruiz-Morales, Jorge

Rusu, Alina Simona

Rusu, Diana

Rutecka-Góra, Joanna

Rutkauskaite, Renata

Sacramento, Octávio

Sadi, Gabriel

Safta, Adela Sorinela

Sagone, Elisabetta

Sahar, Arif

Sainton, Jean-Pierre

Salonen, Tapio

Sánchez Rodríguez, Virginia

Sánchez Vera, Fulgencio

Sánchez-Barroso, Gonzalo

Sánchez-Prada, Andrés

Šandrk Nukić, Ivana

Sandy, Larissa

Sani, Ana

Santha, Agnes
Sastre-Merino, Susana

Sauer, Carsten

Savolainen, Iina

Sazonov, Vladimir

Schaffnit, Susan

Schelleman-Offermans, Karen

Scherer, Lexie

Schmalzbauer, Leah

Schmidt, Eva-Maria

Schnettler, Sebastian

Schultz, David

Schulz, Jay

Schumm, Walter R.

Seabra, Filipa

Segal, Uma

Shacham, Maayan

Sharples, Rachel

Shaver, Frances M.

Shepherd, Andrew

Shim, Jae-Mahn

Shishkina, Alisa

Shlapentokh, Dmitry

Sikirić, Ana Marija

Silva, Eric

Silva, Rui

Silva, Susana

Simão, Cláudia

Simeon, James C.

Simonelli, Andrea

Siriwardane, Rapti

Sitar-Tăut, Dan-Andrei

So, Wi-Young

Sobantu, Mzwandile

Sobrino-Garcia, Itziar

Sofianidis, Angelos

Solano Pinto, Natalia

Solarz, Anna

Sousa, Marlene Filipa Da Natividade E.

Sousa, Nuno

Spacek, Miroslav

Sparaci, Laura

St. John, Tanya

Stahler-Sholk, Richard

Staines, Jo

Staples, Kelly

Stefanik, Miroslav

Steglich, Elissa C.

Stephenson, Max O.

Stinson, Philip M.

Stončikaitè, Ieva

Storhaug, Anita Skårstad 
Straiton, Melanie L.

Strielkowski, Wadim

Strohl, Jeff

Stulp, Gert

Sundström, Gerdt

Supervía, Pablo Usán

Suvi, Heikkinen

Swales, Bridgitte

Sylvia, J. J., IV

Sypion-Dutkowska, Natalia

Szopińska, Kinga

Szóstak, Mariusz

Sztando, Andrzej

Szymaniec, Piotr

Tajdin, Mustapha

Talukder, Byomkesh

Taylor, Abigail

Tchumkam, Herve

Teixeira Vale, Vera

Teixeira, Carlos

Thai, Yvonne

Thomas, Roger E.

Thompson, Piers

Thurston, Chloe

Tidwell, Tawni

Tiziana, Mancini

Tomczyk, Łukasz

Topa, Gabriela

Tragaki, Alexandra

Tran, Van C.

Trein, Philipp

Trifunović, Aleksandar

Trujillo-Torres, Juan M.

Tsai, Fu-Sheng

Tselebis, Athanasios

Tsotsos, Lia E.

Tunio, Muhammad Nawaz

Umland, Andreas

Unanue, Maria Concepción

Undurraga, Rosario

Upton, Geoffrey

Urbanek, Arkadiusz

Urbano, Claudia

Vaccarelli, Alessandro

Valackienè, Asta

Valderrama-Hernández, Rocío

Van Engeland, Anicee

Vasilevski, Vidanka

Vázquez-Parra, José Carlos

Veale, Angela

Velicogna, Marco
Venditto, Bruno

Vera-Valdés, J. Eduardo

Verdicchio, Mario

Vergara, Diego

Vergou, Pinelopi

Vese, Donato

Vieten, Ulrike M.

Vik, Tennley

Viñas, Ana Isabel

Vintila, Mona

Virlanuta, Florina

Visvizi, Anna

Vitale, Tommaso

Vlajčić, Davor

Voroshilova, Anzhelika

Vuolajärvi, Niina

Wadley, Susan Snow

Wakeel, Fathima

Walsh, Peter William

Wang, Han-I

Wang, Zhuoying

Wasileski, Gabriela

Weatherburn, Amy

Weber, Leanne

Weisz, Carolyn

Wettstein, Martin

Wildcat, Matthew

Willführ, Kai

Wilmott, Dominic

Windisch, Steven

Windsong, Elena

Winking, Jeffrey

Winkle, Melissa

Wischmann, Anke

Wolniak, Radosław

Wosiek, Małgorzata

Wroblewski, Angela

Wydra, Doris

Wysocki, Jacek

Wysocki, Marian

$\mathrm{Xu}$, Xiaohe

$\mathrm{Xu}$, Yanfeng

Yang, Liu (Lydia)

Yang, Xiaoli

Lo, Wing Yee

Yoopetch, Chanin

Yu, Yeong Jung

Zachorowska-Mazurkiewicz, Anna

Zajda, Joseph

Zambrana, Ivis Garcia

Zare, Hossein 
Zepeda, Susy

Zhang, Hongzhi

Zierer, Klaus
Ziff, Anna

Zolnikov, Tara Rava

Zulauf-McCurdy, Courtney 